\title{
Reflexiones en torno a la vinculación de la educación y el género
}

\section{Reflections on Education and Gender}

\author{
Ileana Vargas Jiménez ${ }^{1}$ \\ Centro de Investigación y Docencia en Educación \\ Universidad Nacional \\ Heredia, Costa Rica \\ vargasileana@hotmail.com
}

Recibido 18 de enero de 2011 • Aceptado 09 de marzo de 2011

\begin{abstract}
Resumen. El siguiente artículo plantea una reflexión acerca de cómo la educación, desde la escuela, propicia, a partir del currículo oficial y del currículo oculto, una serie de elementos que tienden a hacer diferencias en cuanto a género. Además, se plantean una serie de aspectos tales como la televisión, las prácticas pedagógicas de los grupos de docentes, que inciden directamente en la discriminación sexista. Se muestran, también, algunas recomendaciones que aportan de investigadores para contrarrestar la discriminación por género. Finalmente se presentan las conclusiones.
\end{abstract}

Palabras claves. Educación, género, currículo, co educación, escuela, discriminación.

Abstract. This paper provides an analysis on how education, since the first grades and based on the official or a hidden curriculum, favors several elements that tend to create differences in terms of gender. The study presents different aspects, such as television programs and some teaching practices, that have a direct effect on gender discrimination. There are also recommendations on how to counteract gender discrimination, and conclusions are provided in the last section.

Keywords. Education, school, curriculum, gender, discrimination.

\section{Introducción}

El género es un tema que está cobrando cada vez más debate y análisis, especialmente en la educación, por lo que el presente ensayo analiza aspectos relacionados con la escuela como microcosmos social. Señala a quienes inician el debate de mirar con un nuevo rostro la escuela. Analiza el papel de los medios de comunicación en cuanto a género. Posteriormente, muestra las distintas formas de discriminación, y cómo la universidad, la escuela y el colegio se encuentra estrechamente vinculados con la discriminación de género, lo cual es ratificado por diversos autoras y autores consultados para la realización del presente ensayo.

Otro punto de reflexión es el de la formación docente y la co educación, es decir, cómo se pueden generar cambios sustanciales, pero sobre todo crear conciencia en la necesidad de cambiar aptitudes, modelos de enseñanza, prácticas pedagógicas de docentes, entre otros aspectos.

\footnotetext{
Licenciada en Educación Preescolar, con una maestría en Administración Educativa por la Universidad de New México, Estados Unidos. Se ha desempeñado como directora, investigadora y académica de la División de Educación para el Trabajo del Centro de Investigación y Docencia en Educación (CIDE) de la Universidad Nacional, Costa Rica. Decana del Centro de Investigación y Docencia en Educación (CIDE) de la Universidad Nacional, Costa Rica.
} 
Finalmente, se plantea el tema del género como contenido transversal en educación, esto es, cómo incorporarlo desde del currículo, libros de texto, metodologías de clase, entre otros y vincularlo a una educación igualitaria entre hombres y mujeres, en donde no exista desigualdad.

\section{La escuela como microcosmos social}

Es fundamental precisar, en primer término, qué es la escuela, en vista de que es ahí de donde parte este análisis. Según Pérez (2000):

(...) la escuela como un espacio ecológico de cruce de culturas cuya responsabilidad específica, que la distingue de otras instituciones e instancias de socialización y le confiere su propia identidad y su relativa autonomía, es la mediación reflexiva de aquellos influjos plurales que las diferentes culturas ejercen de forma permanente sobre las nuevas generaciones para facilitar su desarrollo educativo (...). (p. 17)

Lo citado significa que los responsables definitivos de lo que aprenden los alumnos y alumnas en su vida escolar están alojadas en las disciplinas científicas, artísticas, y filosóficas; el currículo; la cultura escolar, constituida por los valores, presentes en los roles, normas, rutinas y ritos propios de la escuela como institución específica; así como las experiencias adquiridas por cada estudiante a través de los intercambios en su entorno.

\section{¿Quiénes inician con el debate de mirar con un nuevo rostro a la escuela?}

En este apartado, Subirats (1999) realiza su análisis recalcando que tanto la escuela como los seres humanos tienen un destino marcado: si eran hombres, debían comportarse como tales; si eran mujeres, debían realizar las tares domésticas y parir hijos. La escuela tenía su tarea asignada: la de transmitir a las nuevas generaciones los comportamientos considerados legítimos.

Sin embargo, de acuerdo con Subirats (1999), eso está cambiando, especialmente porque:

(...) en el caso concreto de la escuela se inician una serie de cuestionamientos sobre las influencias no manifiestas que no abarcan solamente a los conocimientos sino al establecimiento de identidades, jerarquías y desigualdades así como se empieza a tratar de cambiar la escuela para que no contribuya a transmitir estereotipos que limiten el desarrollo de la personalidad humana. (p. 19)

Por otro lado, pareciera que existen más coincidencias que divergencias entre las investigaciones leídas. Tal es el caso de Araya (2004), quien aboga por una enseñanza no sexista, en donde la educación debe mirar los roles docentes y el currículo, procurando la igualdad de género. Igualmente (Acker, 2003) realiza todo un análisis desde la investigación cuantitativa y demuestra que, aunque las mujeres han incursionado en nuevos campos laborales, todavía existen serias diferencias entre los hombres y las mujeres en el ámbito social. Por esto la autora aboga para mantener la independencia y originalidad de los estudios de la mujer y la pedagogía feminista en donde el género sea la idea central.

En tal sentido, coinciden Fioretti, Tejero y Díaz (2002) junto con su equipo de investigadores desde Argentina y por otro lado Ketterer (2008), con sus hallazgos desde Chile: encuentran que los 
maestros, especialmente los pertenecientes a comunidades rurales, estimulan todo lo relacionado a género masculino, en el sentido de que se sigue favoreciendo la participación masculina dentro del salón de clases; así mismo, en las preguntas que, en el salón de clases, elaboran sus docentes, resulta evidente que se da prioridad a la participación de los varones y eso se percibe tan natural como vivir o respirar. Aunque la incorporación de género se encuentra estipulada en las políticas curriculares, no hay una clara conciencia de ello. Es decir, las niñas están destinadas a su rol de mujer, a ser madre, ama de casa; y el varón tiene que estudiar y tener una carrera.

Por otro lado, están las investigaciones que se hicieron desde las universidades y ahí sobresalen Fioretti et al. (2002) y Graña (2008), quienes encontraron que el género femenino está totalmente invisibilizado; además, desde el hogar, se dan diferencias sustanciales entre ambos géneros, hecho que se refuerza, luego, en el preescolar, con las conductas de los docentes, que apoyan, inconscientemente, todo lo que es sexismo. Subirats y Brullet (1999) efectúan un análisis en donde lo esencial de rescatar y de denunciar, es que se dan diferencias sustanciales entre los géneros desde la familia, la escuela, y esto se reproduce de manera sistemática.

Tomé (1999), junto con un equipo de investigadoras, realizaron una investigación en España con la idea principal de tratar de cambiar las prácticas pedagógicas, desde el salón de clase. Además trabajaron con algunos libros de texto, así como con observación de conductas de docentes participantes y llegaron a la conclusión de que deben darse cambios en torno al género; pero no solo en la escuela, sino que también en la sociedad, especialmente, en las familias, ya que es en donde se establecen las diferencias de género. Esta no es una tarea sencilla, para ello este grupo de investigadoras proponen un modelo denominado: Coeducación, en procura de un cambio radical desde la escuela.

Según Subirats (1999) surgen dos corrientes de pensamiento:

1. La sociología de la educación, que define el sistema educativo como un espacio investido por el poder, carente de neutralidad y, por lo tanto, creador y legitimador de identidades sociales jerarquizadas.

2. El pensamiento feminista, que procura buscar estrategias de liberación.

A continuación, se analizan ambas corrientes: la primera se inicia en los años setenta, con un análisis acerca de la escuela, ya que Bourdieu y Bernstein (citados por Subirats, 1999) comienzan a analizar el sistema educativo como no neutral, pues consideran que ese es uno más de los escenarios en donde los grupos sociales se enfrentan y luchan por los recursos y el poder. Pero, ese poder se produce de un modo especial, debido a que, a través del control de las formas de cultura y de las reglas que definen el funcionamiento de las instituciones, cada grupo social trata de maximizar sus posibilidades en el sistema educativo, es decir,

la escuela tiende a reproducir el sistema social existente y las posiciones de poder y de jerarquía vigentes en cada sociedad (...) [por lo que no debe de extrañar que los estudiantes] (...) procedentes de otros grupos sociales se encuentran en un territorio desconocido y a menudo hostil en relación con su cultura inicial por lo que su fracaso escolar se halla en cierto modo programado. (Subirats, 1999, p. 21)

En relación con esta tesis, las escuelas modernas se han planteado como sistemas neutros que no establecen ninguna diferencia de clase, de sexo o de raza. No obstante, de acuerdo con las lecturas efectuadas, se podría afirmar que eso no es cierto, que efectivamente los sistemas educativos no 
son neutros. Esto es por el currículo oficial y el currículo oculto, en donde el docente, de manera absolutamente inconsciente, elabora diferencias entre géneros $\mathrm{y}$, si se revisan los planes de estudio, probablemente se encuentren diferencias cuando se menciona lo masculino y lo femenino.

Con respecto al segundo punto, el pensamiento feminista, se introduce una distinción fundamental para analizar por separado los comportamientos sociales y los condicionantes naturales vinculados a la condición sexual, es decir, la distinción teórica entre sexo y género. Sostiene además Subirats que

(...) los seres humanos no se constituyen como mujeres u hombres únicamente en función de su sexo, esta es la base de biológica; al contrario, sobre esta base se construye la identidad genérica, que tiene una naturaleza de carácter social: las sociedades han creado sistemas de roles y patrones de comportamiento distinto para hombres y mujeres, es decir, prescripciones sobre lo que deben hacer y sobre lo que deben actuar, en función de su sexo. (Subirats, 1999, p. 23)

En este punto del análisis, vale la pena anotar que Araya (2004) menciona elementos totalmente acordes con los planteados por Subirats(1999), por ejemplo, que la diferenciación sexista afecta a las mujeres y a los hombres, aunque la diferencia es que en una escala jerárquica las mujeres son inferiores.

Según Araya (2004), es posible erradicar el sexismo en la educación, -pero eso es algo que se abordará más adelante en esta investigación-; es necesario reconocer según esta investigadora que:

(...) la discriminación, de la desigualdad y de la segregación [son] problemas educativos. [Y una de las posibles soluciones está en la] educación emancipadora y liberadora comprometida con la superación de las desigualdades sociales (...). (p. 2)

La perspectiva de género es, entonces, un compromiso teórico y político (...) implica por tanto, una acción directa sobre la sociedad para transformar los términos de las relaciones sociales así como una voluntad política para cambiar dichos términos de manera que sea posible la equidad y la igualdad. (pp. 3-4)

Subirats (1999) defiende que lo importante es qué se puede hacer para que la escuela deje de ser sexista y discriminatoria para las mujeres; en tal sentido, inicia con una intensa revisión de características curriculares, de los libros de texto, de las variables contextuales, del lenguaje, del currículo oculto, es decir, de todo aquello que esté regido. según la autora, por una ideología sexista y que contribuye, por tanto, a una socialización diferencial que perjudica a las niñas.

Otra postura desde la visión de Norteamérica planteada por Salomone (2007), plantea un análisis desde el tema de la enseñanza diferenciada por sexos en los Estados Unidos, política educativa y práctica educativa. Existe todo un debate teórico entre los que defienden la enseñanza diferenciada. Este debate es el motivo que genera mayor división en la búsqueda de la igualdad de género en la enseñanza.

Así, de acuerdo con Salomone (2007), los conservadores defienden la educación diferenciada porque se basa en los valores tradicionales y porque apela a que los padres de familia puedan elegir (mercado de diversidad educativa); solicitan que la igualdad educativa sea otorgada a las niñas, para procurar una mejoría en el rendimiento académico global y para favorecer el desarrollo del interés y la competencia en Matemáticas, Ciencias, y Tecnologías, también, el aumento de la autoestima, el aumento de la preferencia por las profesiones tradicionalmente dominadas por los hombres y el aumento de la participación femenina. 
Por otro lado, están los que abogan por la enseñanza no diferenciada, quienes sugieren una intención oculta en el programa institucionalizado para conseguir el domino masculino en la interacción de la clase y que las actitudes institucionalizadas preparen a los estudiantes para un papel social determinado por el género.

En Estados Unidos, no cabe la menor duda, existe un problema de género que se mezcla con el de la raza, la cultura y la clase social. De acuerdo con Salomone (2007), se presentan posturas radicales en cuanto al género, las cuales son totalmente avaladas por la sociedad norteamericana y se ratifica cómo la raza, la diversidad étnica, y las clases sociales son contempladas en la mención de género.

En resumen, el dilema planteado es la homogeneidad, la diferencia y las cuestiones implícitas en relación con la igualdad de género, así como los programas de estudio diferenciados por sexos, en los cuales se forman dos bandos donde cada uno apoya una propuesta. Esto indica y, queda claramente mencionado, que la discriminación es un factor determinante cuando se habla de género, y cómo en la sociedad norteamericana se enfatiza de manera solapada el hecho de la igualdad de género. La sociedad juega un papel preponderante, ya que da las pautas acerca de cómo se marcan las diferencias, por raza, por religión, por idioma, por nivel educativo. Es así como se aboga por una educación que, en el fondo, lo que plantea es favorecer al sexo masculino, aunque se crea o diga que se quiere la igualdad.

\section{¿Qué papel juegan los medios de comunicación en cuanto a género?}

En este análisis es necesario mencionar la influencia que tienen los medios de comunicación masiva en los jóvenes, niños y niñas, especialmente porque son medios que reproducen las ideologías aceptadas por la sociedad y, por ende, por la familia. Lo lamentable es que, según investigaciones realizadas, los estereotipos que reproducen en cuanto al género son asombrosamente sorprendentes. En ese sentido, Belmonte y Guillamón (2008) efectúan un análisis muy particular con respecto a los estereotipos de género en la televisión, resaltando el hecho de "co educar la mirada".

La televisión, al ser un medio masivo y de tanto alcance social, está implicada en la construcción de los significados, es decir:

(...) Los productos televisivos contribuyen, de forma cotidiana y más o menos sistemática a generar identidades a partir de los mecanismos narrativos, semióticos e interpretativos que se ponen en marcha en cada acto de significación. (Belmonte y Guillamón, 2008, p. 116)

En otras palabras, la ideología de género se transmite.

¿Cómo afecta la televisión a los jóvenes, niños y niñas? Resulta particularmente significativo cómo el universo mediático ayuda a la construcción de identidades desde la más tierna infancia, así como en la adolescencia, fases consideradas como cruciales para el desarrollo humano.

Otro punto importante es que los estereotipos se definen como un conjunto de ideas acerca de los géneros, ideas que favorecen el establecimiento de roles fuertemente arraigados en la sociedad.

Por lo anterior, algunas series de televisión, según Belmonte y Guillamon (2008): “(...) presentan un discurso normalizador sobre usos, costumbres, roles y relaciones de género que ayudan a reforzar los estereotipos sociales (...)" (p. 117).

Lo que a la postre significa es que se ratifica la presencia de estereotipos de género que contribuyen al reforzamiento de la desigualdad. 
Por ende, se podría mencionar con igual preocupación, que uno de los rasgos de la sociedad patriarcal es imaginar la dicotomía como una característica profunda separatista tanto del hombre como a de la mujer y los discrimina en dos clases.

Los autores proponen:

(...) una deconstrucción y análisis crítico de los discursos televisivos (...)” (p. 120). Además, de (...) "“una alfabetización audiovisual’ que enseña a reflexionar sobre (...) los productos mediáticos, y un enfoque desde el género y la coeducación tanto en los ámbitos familiares como escolares (...). (Belmonte y Guillamón, 2008, p. 120)

De acuerdo con los planteamientos analizados, se confirma que los programas televisivos reconocen y permean en las sociedades y en las familias, lo que supone que se acepta, sin cuestionar, si eso que se está viendo es bueno, malo o simplemente ni bueno ni malo. Se reafirma que la televisión, y por ende los medios de comunicación, son piezas fundamentales en todo lo concerniente al género. En el caso costarricense se podrían mencionar, por ejemplo, la serie "Familia Simpson" donde es evidente la imagen patriarcal, así como la imagen de la mujer como ama de casa.

\section{La expresión de las distintas formas de discriminación}

En ese sentido, vale la pena señalar varios estudios que se efectuaron especialmente en América del Sur, en donde se encontraron hallazgos muy interesantes, por ejemplo en Uruguay. Al respecto, Graña (2008, p. 78) dice:

(...) el género es un complejo haz de conductas, orientaciones de carácter, posturas corporales, actitudes y preferencias, que aprehendemos por las vías multiformes de la experiencia de vida, (...) se fusiona con el sexo biológico, (...) el género (social, aprendido) se nos presenta a nuestra autopercepción como si fuera inseparable del sexo (biológico, heredado) (...)

El autor llega a cinco conclusiones: la primera es que el aprendizaje del género se inicia desde la primera infancia, con la familia y luego en el preescolar. La segunda es que dicho aprendizaje continua luego en el ciclo escolar, en donde solapadamente, bajo la filosofía de la igualdad, se reproducen, por medio de los textos escolares dominantes en la escuela. En esto coincide con Subirats (1999). Una tercera conclusión son las paradojas de los docentes y las docentes con sus intervenciones, comportamientos e inclinaciones con respecto al género; la cuarta conclusión es que se comprueba la invisibilización de la mujer y un fuerte dominio androcentrista de la racionalidad occidental y, por último, la conducta de los estudiantes es atribuible a una baja auto-estima (Graña 2008).

Lo que llama la atención de su análisis es que las mujeres creen que su desempeño en el colegio se debe a su esfuerzo y no a su capacidad, muy diferente de los varones, quienes perciben que su desempeño se debe a sus capacidades. Esta posición coincide con Subirats (1999 y 1994) y Tomé (1999) cuando afirman que los varones se hacen frecuentemente más visibles en sus manifestaciones en el aula respecto a las niñas.

Otro punto coincidente con Tomé (1999) es que las alumnas atribuyen sus dificultades en Matemáticas a su falta de capacidad personal: ellas perciben que el principal recurso puesto en juego por los varones para obtener mejores resultados académicos es la inteligencia y el de las mujeres es la aplicación al estudio. Esto es reafirmado por Salomone (2007) Subirats $(1999,1994)$, 
Subirats y Brullet (1999). Por lo que se puede aceptar que desde que se nace se impone, desde la familia, el aprendizaje con respecto al género y, luego, la escuela lo reafirma tanto con el currículo como con las aptitudes de los docentes encargados de educar en igualdad de condiciones; sin embargo, lo anterior se da en la mayoría de los países, según los artículos consultados para este ensayo.

En la siguiente figura se explicita lo anotado, especialmente las cuatro grandes áreas de estudio: la familia, la escuela, la sociedad y la iglesia. El género está permeado por todas ellas. Igualmente se incorporaron los aspectos más importantes en cada una de las áreas.

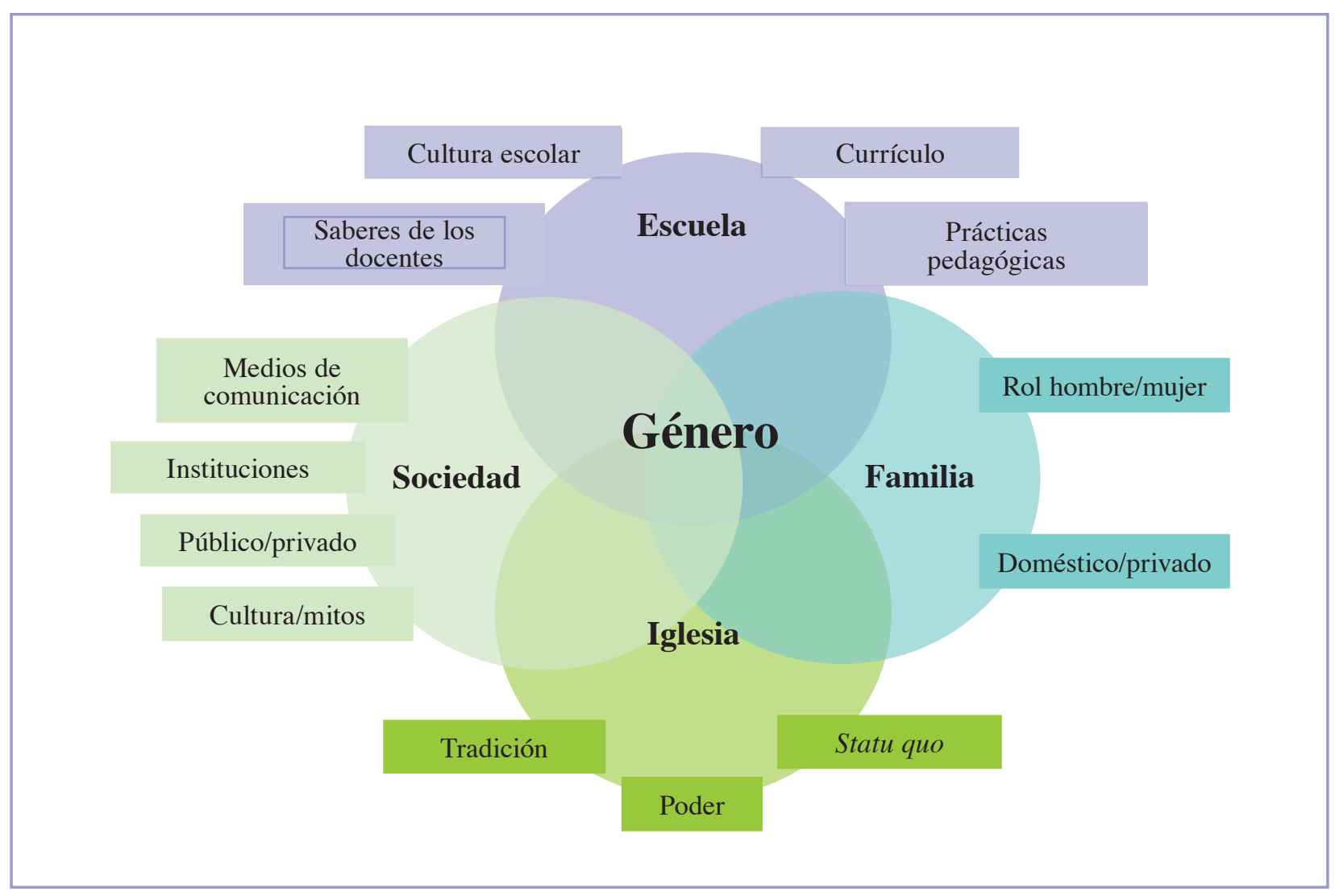

Figura 1. Aspectos de la sociedad, escuela, familia e iglesia que influyen en la categoría género.

Nota. Elaboración propia.

\section{¿Se pueden justificar estos hallazgos, en el sentido de que el trato de los estudiantes y las estudiantes dentro del salón de clases es desigual?}

Según Subirats y Brullet (1999), las investigaciones que se han llevado a cabo acerca de las interacciones dentro del aula buscan respuestas de cómo el trato desigual es cierto. El análisis de la relación entre maestros y maestras y alumnos y alumnas muestra que los niños reciben mayor desaprobación y más críticas de su maestros que las niñas y que las críticas a los niños adoptan un tono de voz más duro, mientras que las críticas a las niñas se hacen en tono más suave (Gates y MacCoby, citados por Subirats y Brullet, 1999, p. 200).

Estas conclusiones fueron realizadas en los años sesenta y demuestran que en contexto costarricense es prácticamente igual: los niños reciben una mayor desaprobación por parte de sus maestros y maestras y cuando se les llama la atención a las niñas, se hace de una manera mucho más suave, lo que conlleva a que se den diferencias en el trato entre hombres y mujeres, como ya se ha manifestado. 


\section{La formación docente y la co educación ${ }^{1}$}

En este apartado del ensayo se hace necesario plantear cómo se pueden generar cambios desde la formación docente, lo cual no es una tarea fácil, ya que se tienen que generar poco a poco transformaciones desde los planes de formación docente, así mismo se deben contemplar políticas desde las unidades académicas ${ }^{2}$, de igual manera crear la cultura de igualdad de género, incorporando a los académicos y académicas desde la formulación de planes, en donde se evidencie la necesidad de introducir cambios que lleven a una educación no sexista.

Sin dejar de recordar que se han adquirido actitudes, aptitudes, rasgos, conductas, que las personas mismas ignoran y, por ende, las proyectan haciendo distinción de género. Esto es precisamente, lo que se debe cambiar: la forma de conducir la clase, las prácticas pedagógicas utilizadas, versus saberes de docentes, el lenguaje utilizado, el currículo oficial, y el currículo oculto, entre otros.

Vale la pena señalar tres puntos importantes que plantea Subirats (1999), a pesar que lo hace desde la escuela, igual se da en las universidades:

1. Lo primero que debe darse es un mayor protagonismo y una mayor visibilidad a las niñas en la escuela, lo que trae como resultado un aumento del éxito escolar femenino. Como consecuencia de lo anterior, indudablemente, el movimiento co educativo ha hecho emerger problemas, ya que se cree que lo que se quiere es favorecer a las mujeres, lo que ha ocasionado, por supuesto, ciertas resistencias por parte de los profesores y alumnos. Al respecto es atinente recordar que todo cambio genera resistencia, pero sobre todo temor y miedo, especialmente si eso tiene que ver con educar en igualdad.

2. La co educación supone modelos de enseñanza que afectan tanto la educación de niños como la de las niñas. Se insiste en que lo que se debe de encontrar es una búsqueda mayor de igualdad en contra de las diferencias.

3. Las capacidades y aptitudes atribuidas a las mujeres y a los hombres varían de una sociedad a otra, de una época a otra, lo que quiere decir es que esas capacidades y aptitudes no están atribuidas a la biología sino a lo social, y eso no cabe duda que afecta, pero positivamente, para las mujeres, al no tener que demostrar ante los hombres que ellas tienen capacidades superiores.

Por otro lado, Acker (2003), Fioretti, et.al. (2002), Graña (2008) y Subirats (1999) concuerdan en que las mujeres, desde las universidades, sufren discriminación por su género, y no solamente eso, sino que se evidencian ciertas conductas en los varones al tener ellos los puestos de mayor envergadura en las universidades, donde las mujeres no tienen las mismas oportunidades, por su condición de género.

\section{La transversalización de género en educación}

La transversalización es vista en este análisis como el eje integrador que traspasa a todo el plan de estudios, es decir, al currículo, por lo tanto debe permear todo el quehacer del docente, llámese escuela, colegio o universidad, de ahí la necesidad de mencionarlo.

En nuestro contexto educativo el término co educación no es utilizado.

Se define la unidad académica como la instancia que desarrollan carreras, proyectos de investigación, extensión, compuesta por administrativos, académicos y académicas, y los estudiantes. 
En ese sentido, "transversalidad de género es un enfoque transformador, que apunta a analizar los impactos diferenciados del sistema de género entre hombres y mujeres, que permite tener en cuenta las especificidades de las personas como seres integrales (...) (UNAGaceta, 2010, p. 8).

En el caso concreto de la Universidad Nacional, localizada en Heredia, Costa Rica se ha optado por una serie de Políticas para la Igualdad y Equidad de Género, (UNA-Gaceta, 2010, p. 8).

La pregunta que surge es la siguiente: ¿es posible cambiar, sobre todo generar cambios desde la educación? En este espacio, la igualdad, entendida como las mismas posibilidades y oportunidades en el salón de clases para mujeres y varones, sin distinción de género, es el ideal que se debe tratar de lograr. En el caso concreto de Costa Rica se pueden iniciar cambios tales como los siguientes:

Se debe partir de un currículo emergente, en donde el docente no puede ser un simple espectador o espectadora, debe aplicar las estrategias y rutinas aprendidas en los años de formación académica, debe convertirse en un investigador de aula, según lo plantean Subirats $(1994,1999)$ y Tomé (1999), por cuanto en sus investigaciones uno de sus más sobresalientes hallazgos es precisamente el hecho de que se debe efectuar investigación acción desde el aula.

Además, ese docente se encuentra en su ámbito natural donde desarrolla su práctica, ámbito donde aparecen los problemas y donde deben experimentarse estrategias de intervención acordes con el contexto y la situación, de manera que converjan el respeto, la diversidad y la solidaridad entre los estudiantes y las estudiantes.

\section{Conclusiones}

Una de las conclusiones a las que se llega es que en la escuela se articulan, fomentan y reproducen las desigualdades de género. Se confirma que en Argentina, Chile, Estados Unidos, Uruguay, España, y Costa Rica se comparte un mismo destino, en donde la enseñanza es vista desde la perspectiva masculina, puesto que las docentes y los docentes reproducen los modelos de educación heredados de generación en generación, lo anterior es asumido y considerado como algo sencillamente normal.

Se establece que se continúa coadyuvando a promover la desigualdad en cuanto a género desde la familia, la escuela, el colegio, los medios de comunicación, la iglesia y las universidades.

Así mismo, se deben generar cambios sustanciales realizando investigaciones, desde las universidades, que promuevan en todo momento la igualdad de género. Además es necesario que se incorpore, en los planes de estudio, el tema de género y cómo puede ser abordado desde el salón de clases; otra tarea que se puede señalar es la referida al currículo universitario, en el sentido de que se debe plasmar el estudio de género como eje transversal.

Esencial es que, desde la educación, específicamente desde la escuela, colegio y universidad se establezcan políticas claras y participativas que coadyuven a lograr una educación no sexista, generadora de la no discriminación; todo lo anterior de la mano con la familia, ya que es el agente socializador que debe procurar romper con las barreras sexistas.

Finalmente, los medios de comunicación deben replantear las formas de reproducir programas de televisión que generan en la mayoría de las ocasiones desintegraciones de los géneros, lo que provoca las divisiones entre sexo y remarcan que eso es algo natural, normal, y aceptado por todos los miembros de la sociedad. 


\section{Referencias bibliográficas}

Acker, S. (2003). Género y educación. Reflexiones sociológicas sobre mujeres, enseñanza y feminismo. España: Narcea, S. A. de Ediciones.

Araya, S. (2004). Hacia una educación no sexista. Revista Electrónica Actualidades Investigativas en educación 4(2), 1-13. Recuperado de http://revista.inie.ucr.ac.cr/articulos/2-2004/ archivos/sexista.pdf

Belmonte, J. y Guillamón, S. (2008). Co-educar la mirada contra los estereotipos de género en TV. Comunicar, 16(31), 115-120. Recuperado de http://redalyc.uaemex.mx/pdf/158/15803115.pdf

Fioretti, S., Tejero, G. y Díaz, P. (2002, Segunda época). El género: ¿un enfoque ausente en la formación docente? La Aljaba, Año/Vol. 7, 171-186. Recuperado de http://redalyc. uaemex.mx/pdf/278/27800708.pdf

Graña, F. (2008). El asalto de las mujeres a las carreras universitarias "masculinas": cambio y continuidad en la discriminación de género. Praxis Educativa, 12, 77-86. Recuperado de http:// redalyc.uaemex.mx/pdf/1531/153112902008.pdf

Ketterer, L. (2008). La transversalización de género en la educación: ¿Qué pasa en las escuelas de Galvarino, la capital indígena de la región de La Araucanía? La Aljaba. Segunda época, 12, 21-32. Recuperado de http://www.scielo.org.ar/pdf/aljaba/v12/v12a02.pdf

Pérez, A. (2000). La cultura escolar en la sociedad neoliberal ( $3^{\mathrm{a}}$ ed.). España: Ediciones Morata, S. L.

Salomone, R. (2007, setiembre-diciembre). Igualdad y diferencia. La cuestión de la equidad de género en la educación. Revista española de Pedagogía, 65(238), 433-446. Recuperado de http:// dialnet.unirioja.es/servlet/articulo?codigo $=2576686$

Subirats, M. (1999). Género y escuela. En C. Lomas (Coord.). ¿Iguales o diferentes? Género, diferencia sexual, lenguaje y educación (pp. 19-32). España: Paidós Ibérica.

Subirats, M. y Brullet, C. (1999). Rosa y azul. La trasmisión de los géneros en la escuela mixta. En M. Belausteguigoitia y A. Mingo (Coord.), Géneros prófugos. feminismo y educación (pp. 189-224). México: Paidós.

Subirats, M. (1994, septiembre-diciembre). Conquistar la igualdad: la coeducación hoy. Revista Iberoamericana de Educación, 6, 49-78. Recuperado de http://www.rieoei.org/ oeivirt/rie06a02.htm

Tomé, A. (1999). Un camino hacia la coeducación (instrumentos de reflexión e intervención). En C. Lomas (Coord.). ¿Iguales o diferentes? Género, diferencia sexual, lenguaje y educación (pp. 171-198). España: Paidós. 
UNA-GACETA 09-2010. (15 de junio de 2010). Política para la igualdad y equidad de género en la Universidad Nacional [SCU-988-2010, ARTÍCULO VIII, INCISO IV, de la sesión ordinaria celebrada el 20 de mayo del 2010, acta No. 3076], pp. 6-10. Heredia, Costa Rica. Recuperado de http://www.juridica.una.ac.cr/index.php?option=com remository\&Itemid=0\&func=startdo $\underline{\text { wn\&id }=581}$ 